\title{
Medidas lineares do limbo foliar dos capins xaraés e massai para a estimativa da área foliar
}

\author{
Wilton Ladeira da Silva \\ João Paulo Ramos Costa * \\ Gregory de Paula Caputti \\ Leandro Galzerano \\ Ana Cláudia Ruggieri \\ Universidade Estadual Paulista, Faculdade de Ciências Agrárias e Veterinárias \\ Via de acesso Prof. Paulo Donato Castellane, s/n, CEP 14884-900, Jaboticabal - SP, Brasil \\ * Autor para correspondência \\ jpramoscosta@hotmail.com
}

Submetido em 22/10/2012

Aceito para publicação em 02/04/2013

\section{Resumo}

O conhecimento da área foliar de gramíneas forrageiras é fundamental, já que é uma das mais importantes variáveis na avaliação do crescimento vegetal. Assim, objetivou-se determinar equações que possibilitem, por meio de medidas simples de comprimento, larguras média e máxima das folhas, estimar de maneira rápida e precisa a área foliar real de Brachiaria brizantha cv. Xaráes e de Panicum maximum cv. Massai. Foram tomadas com réguas milimetradas as medidas de comprimento ao longo da nervura principal $(\mathrm{C})$, largura máxima perpendicular à nervura principal $\left(\mathrm{L}_{\text {máx }}\right)$ e largura média $\left(\mathrm{L}_{\text {méd }}\right)$ dos limbos foliares das duas espécies. Para determinação das áreas foliares reais (AFR) foi utilizado o integrador Li-Cor ${ }^{\circledR}$, modelo LI 3000. Foram realizados estudos de regressão e correlação entre a AFR e área foliar estimada pelas equações lineares ou exponenciais na escolha das melhores equações. No capim xaraés, a equação com maior precisão que estimou a AFR foi a linear 0,53+0,98 CLmed e no capim massai foi a linear $1,30+0,92 \mathrm{CLmed}$ e as exponenciais $8,86 \mathrm{e}^{0,04 \mathrm{Clmax}}$ e $10,30 \mathrm{e}^{0,03 \mathrm{Clmed}}$. Estimativas de área foliar de capim xaraés e capim massai por meio de medidas simples de comprimento e largura de folhas provaram-se eficientes e precisas.

Palavras-chave: Brachiaria brizantha; Gramíneas forrageiras; Panicum maximum; Pastagens

\section{Abstract}

Linear measurements of the leaf blade in xaraes and massai grasses for estimation of the leaf area. Knowledge on the leaf area of foraging grasses is essential, since it's one of the most important variables in the evaluation of plant growth. Thus, one aimed at determining equations which allow, through simple measurements of leaf length, as well as average and maximum width, to quickly and accurately estimate the actual leaf area of Brachiaria brizantha cv. Xaraes and Panicum maximum cv. Massai. One measured with millimeter rulers the length along the main vein $(\mathrm{L})$, the maximum width perpendicular to the main vein $\left(\mathrm{W}_{\max }\right)$, and the average width $\left(\mathrm{W}_{\text {ave }}\right)$ of leaf blades in both species. For determining the actual leaf areas (ALA), one used the Li-Cor ${ }^{\mathbb{B}}$, model LI 3000. Regression and correlation studies were performed between ALA and the leaf area estimated 
through the linear or exponential equations for choosing the best equations. For xaraes grass the equation with the best accuracy for estimating ALA was the linear $0.53+0.98 \mathrm{LW}_{\text {ave }}$ and for massai grass the best options were the linear $1.30+0.92 \mathrm{LW}_{\text {ave }}$ and the exponential $8.86 \mathrm{e}^{0.04 \mathrm{LWmax}}$ and $10.30 \mathrm{e}^{0.03 \mathrm{LWave}}$. Estimates of the leaf area of xaraes grass and massai grass through simple measurements of leaf length and width have proved to be effective and accurate.

Key words: Brachiaria brizantha; Foraging grasses; Panicum maximum; Pastures

\section{Introdução}

Atualmente, o manejo de pastagens é caracterizado por diversas técnicas que possibilitam ao pecuarista otimizar a produção, seja ela de carne, leite, lã, dentre outros. E como hoje, no Brasil, as pastagens já não são mais formadas exclusivamente por uma única espécie forrageira, o conhecimento das características morfológicas das diferentes espécies torna-se essencial para orientação do manejo destas plantas, uma vez que o manejo deve ser realizado de forma diferenciada de acordo com a espécie forrageira utilizada.

O capim-xaráes [Urochloa brizantha (Hochst. ex A. Rich.) R. Webster] foi estudado e avaliado em conjunto pelas Embrapa Gado de corte e cerrados por mais de 10 anos, até ser lançada em 2002. São plantas cespitosas que podem enraizar nos nós basais; os colmos possuem poucas ramificações; as bainhas possuem pêlos claros; a lâmina foliar pode chegar a $64 \mathrm{~cm}$ de comprimento e $3 \mathrm{~cm}$ de largura, com bordos arroxeados e ásperos. São plantas de fácil estabelecimento, alta produtividade, especialmente de folhas. Apresenta boa resposta à adubação, é tolerante a fungos foliares e de raiz, seu florescimento é intenso, rápido e concentrado no outono (VALLE et al., 2004), possui outras vantagens, como maior velocidade de rebrota e produção de forragem (EUCLIDES et al., 2009).

O capim-massai, originado do cruzamento entre Panicum maximum e Panicum infestum, foi selecionado no Brasil, pela Embrapa Gado de Corte e lançado comercialmente em 2001 (EMBRAPA, 2001). São plantas cespitosas de baixo porte, com média de 0,6 $\mathrm{m}$, com folhas estreitas (em torno de $0,9 \mathrm{~cm}$ ) e eretas, quebrando nas pontas. São plantas que apresentam média pilosidade. As inflorescências são do tipo intermediária entre panícula e racemo. São plantas com exigência média a alta em relação à fertilidade dos solos e possuem boa produção sob pastejo (EMBRAPA, 2001).

Considerando a recente implantação de pastagens no Brasil com estas duas forrageiras, existe uma grande necessidade de estudos básicos que envolvam aspectos relacionados ao manejo destas plantas quando submetidas ao pastejo ou mesmo sob cortes, como a reprodução, o crescimento, desenvolvimento, exigências em nutrientes, persistência na área, e outros. $\mathrm{Na}$ maioria destes estudos, o conhecimento da área foliar é fundamental, já que é uma das mais importantes variáveis na avaliação do crescimento vegetal (BIANCO et al., 2000). Hoje, existem vários métodos destrutivos e não destrutivos, diretos ou indiretos, para se estimar a área foliar, sendo variável a precisão dos mesmos. A utilização de determinado método irá depender da precisão desejada, do tempo gasto para estimar a área foliar, da manutenção das plantas, dentre outros.

A vantagem da utilização do método não destrutivo está na possibilidade de se poder acompanhar o crescimento e a expansão foliar da mesma planta até o final do ciclo ou da avaliação, além de ser rápido e relativamente preciso (BIANCO et al., 2000). Desta forma, a estimativa da área foliar com o uso de variáveis dimensionais de folhas é utilizada para diversas espécies vegetais e em diversas condições (SILVA et al., 2002; ADAMI et al., 2008; BIANCO et al., 2008; MALDANER et al., 2009; CARVALHO et al., 2011; GALZERANO et al., 2012). Assim, a utilização de equações de regressão entre a área foliar real e variáveis dimensionais lineares das folhas é considerado um método não destrutivo amplamente empregado no campo (ADAMI et al., 2008).

Objetivou-se com este trabalho determinar equações que possibilitem, por meio de medidas simples de comprimento e larguras média e máxima das folhas, 
estimarem, de maneira rápida e com boa precisão, a área foliar real dos capins xaráes e massai.

\section{Material e Métodos}

O estudo foi conduzido no setor de Forragicultura pertencente ao Departamento de Zootecnia da Faculdade de Ciências Agrárias e Veterinárias - UNESP, Campus de Jaboticabal, localizado a $21^{\circ} 15^{\prime}$ de latitude sul e $48^{\circ} 18^{\prime}$ de longitude oeste, em altitude de $595 \mathrm{~m}$. O clima, segundo a classificação de Köppen, é do tipo Aw tropical com inverno curto, moderado e seco, verão quente e chuvoso, caracterizando duas estações distintas.

No mês de janeiro de 2011, compreendido na época das "águas", foram coletados de forma aleatória e em diversas posições dos pastos já estabelecidos, aproximadamente 50 perfilhos do capim-xaraés e 50 perfilhos do capim-massai, que foram colocados em caixas de isopor com gelo para se evitar o murchamento das folhas. Na sequência, os perfilhos foram levados ao laboratório para separação do limbo foliar dos colmos.

Foram retirados dos perfilhos 60 limbos foliares de cada espécie, em diferentes posições dos perfilhos. Estes limbos se encontravam nas mais diversas condições ecológicas e ambientais para que pudessem representar de forma mais real possível as condições que estão sujeitas em nível de campo.

Os limbos foliares de cada cultivar foram destacados e numerados de 1 a 60, tomando-se de cada limbo as medidas de comprimento ao longo da nervura principal (C), largura máxima perpendicular à nervura principal $\left(\mathrm{L}_{\text {máx }}\right)$ e largura média $\left(\mathrm{L}_{\text {méd }}\right)$ obtida pela média de seis medidas de largura perpendiculares ao longo do limbo. Estas medições foram feitas com auxílio de uma régua milimetrada.

As áreas foliares reais (AFR) foram determinadas com o auxílio do integrador de área foliar Li-Cor ${ }^{\circledR}$, modelo LI 3000. Os limbos foliares foram passados pelo aparelho, um a um, em ordem numérica, e os valores de AFR anotados em uma planilha.

Estudos de regressão foram realizados para que se pudesse escolher a equação que representasse a área foliar (AF). Estas equações foram divididas em dois tipos, as lineares $(\mathrm{Y}=\mathrm{a}+\mathrm{bx})$ correspondentes às equações de 1 a 5 e as exponenciais $\left(\mathrm{Y}=\mathrm{ae}^{\mathrm{bx}}\right)$ correspondentes às equações de 6 a 10. $\mathrm{O}$ valor $\mathrm{Y}$ estimou a área foliar do limbo foliar em função de $\mathrm{X}$, cujos valores poderiam ser o comprimento $(\mathrm{C})$, a largura máxima $\left(\mathrm{L}_{\text {máx }}\right)$, largura

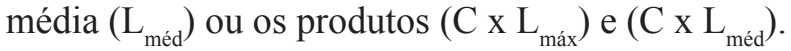

Para a escolha das melhores equações, utilizou-se os seguintes critérios: a) coeficiente de determinação $\left(r^{2} \geq 0,95\right)$; b) coeficiente de correlação $(R)$ entre a área foliar real e os valores estimados pelas equações, sendo adotado valor maior ou igual a 0,95 e c) a soma de quadrado dos resíduos, que é a soma de quadrados da diferença entre os valores observados e os preditos pelos modelos onde o menor valor contribui na escolha da melhor equação.

As estimativas das variáveis dos modelos de regressão e demais cálculos, foram realizados pelos procedimentos Proc Reg, Proc Corr e Proc means do pacote estatístico SAS, versão 9.0 (2002).

\section{Resultados e Discussão}

Os resultados referentes aos estudos de regressão efetuados com as comparações da área foliar real (AFR) e as medidas lineares de comprimento (C), largura máxima $\left(\mathrm{L}_{\max }\right)$ e largura média $\left(\mathrm{L}_{\text {med }}\right)$ do limbo foliar e dos produtos do comprimento pelas larguras máxima $\left(\mathrm{CL}_{\max }\right)$ e média $\left(\mathrm{CL}_{\text {med }}\right)$ dos capins xaraés e massai estão apresentados na Tabela 1. Observa-se que todas as equações apresentadas para o capim-xaraés permitiram obter estimativas satisfatórias da área foliar com coeficientes de determinação $\left(r^{2}\right)$ superiores a 0,65 , enquanto que para o capim-massai estes coeficientes foram em média melhores nos tipos de equações exponenciais, superiores a 0,92 , em relação às equações lineares.

De maneira geral, as equações do tipo exponencial foram as que apresentaram os maiores coeficientes de determinação $\left(\mathrm{r}^{2}\right)$ nas duas gramíneas estudadas, sendo estes valores superiores a 0,95 no capim-xaraés e 0,92 no capim-massai.

Os coeficientes de correlação simples envolvendo as 10 equações ajustadas e a área foliar real dos capins 
xaraés e massai, obtida por meio das medições com a régua encontram-se nas Tabelas 2 e 3, respectivamente. Estes coeficientes foram adotados como critério, além dos valores de $\mathrm{r}^{2}$, na escolha das melhores equações.

Ao analisar o coeficiente de correlação (R) das equações entre a AFR e a área foliar estimada do capim-xaraés, observa-se que os maiores valores de $\mathrm{R}$ foram de 0,998 e 0,991 correspondentes às Eq5 e Eq10 respectivamente, e o menor valor 0,812 correspondente à Eq7. Já no capim-massai, o maior valor de $\mathrm{R}(0,978)$ observado entre a AFR e as equações (Tabela 3 ), foi da Eq5, seguido das Eq10 $(0,965)$ e Eq4 $(0,924)$. Porém a Eq4 apresentou valor de $r^{2}(0,8531)$ inferior aos das Eq5 $(0,9565)$ e $\operatorname{Eq} 10(0,9787)$.
Com base nos valores de $\mathrm{r}^{2}$ e do coeficiente de correlação (r), os modelos que mais se adequaram nas relações entre as dimensões lineares e a área foliar no capim-xaráes foram as equações linear Eq5 $\left(0,53+0,98\right.$ CLmed) e exponenciais Eq6 $\left(26,44 \mathrm{e}^{0,01 \mathrm{C}}\right)$, Eq9 $\left(27,556 \mathrm{e}^{0,01 C L \max }\right)$ e Eq10 $\left(27,17 \mathrm{e}^{0,01 C L m e d}\right)$. Porém, ao observar a soma de quadrados do resíduo destas equações verificou-se que a Eq5 ( ${ }^{2}=0,9951$ e coeficiente de correlação igual a 0,998 ) foi a que apresentou o menor valor $(132,56)$, indicando que o produto das duas dimensões do limbo foliar (comprimento e largura média) nesta cultivar, são as que estimam a área foliar de maneira mais conclusiva, podendo ser melhor visualizada na Figura 1. Bianco et al. (2000) trabalharam com duas espécies de Brachiaria brizantha e também encontraram melhores estimativas da área foliar real em função do produto, comprimento $\mathrm{x}$ largura, do limbo foliar.

TABELA 1: Variáveis dimensionais lineares do limbo foliar, equações de regressão estimadas para obtenção da área foliar real, grau de liberdade (GL), coeficiente de determinação $\left(\mathrm{r}^{2}\right)$ e soma do quadrado do resíduo (S.Q.) obtidas da avaliação dos modelos do capim-xaraés e do capim-massai.

\begin{tabular}{|c|c|c|c|c|c|c|}
\hline Modelo & $\begin{array}{l}\text { Variáveis } \\
\text { lineares* }\end{array}$ & Tipo de equação & $\begin{array}{c}\text { Equação da área foliar } \\
\text { estimada }\end{array}$ & GL & $\mathbf{r}^{2}$ & S.Q. \\
\hline & \multicolumn{6}{|c|}{ Brachiaria brizantha cv. Xaraés } \\
\hline Eq1 & $\mathrm{C}$ & Linear & $-27,27+1,83 \mathrm{C}$ & 58 & 0,8973 & 2762,69 \\
\hline Eq2 & Lmax & Linear & $-62,0+67,04 \mathrm{Lmax}$ & 58 & 0,6531 & 9334,84 \\
\hline Eq3 & Lmed & Linear & $-59,3+96,74$ Lmed & 58 & 0,7778 & 5980,51 \\
\hline Eq4 & CLmax & Linear & $-1,54+0,69$ CLmax & 58 & 0,9640 & 969,50 \\
\hline Eq5 & CLmed & Linear & 0,53+0,98 CLmed & 58 & 0,9951 & 132,56 \\
\hline Eq6 & $\mathrm{C}$ & Exponencial & $26,44 \mathrm{e}^{0,01 C}$ & 58 & 0,9830 & 4240,1 \\
\hline Eq7 & Lmax & Exponencial & $28,80 \mathrm{e}^{0,01 L \max }$ & 58 & 0,9528 & 11757,7 \\
\hline Eq8 & Lmed & Exponencial & $29,92 \mathrm{e}^{0,01 \mathrm{Lmed}}$ & 58 & 0,9607 & 9795,9 \\
\hline Eq9 & CLmax & Exponencial & $27,55 \mathrm{e}^{0,01 C \max }$ & 58 & 0,9835 & 4110,6 \\
\hline \multirow[t]{2}{*}{ Eq10 } & CLmed & Exponencial & $27,17 \mathrm{e}^{0,01 \text { CLmed }}$ & 58 & 0,9886 & 2843,00 \\
\hline & \multicolumn{6}{|c|}{ Panicum maximum cv. Massai } \\
\hline Eq1 & $\mathrm{C}$ & Linear & $0,52+0,38 \mathrm{C}$ & 58 & 0,6101 & 930,21 \\
\hline Eq2 & Lmax & Linear & $0,61+25,24 \mathrm{Lmax}$ & 58 & 0,2069 & 1892,24 \\
\hline Eq3 & Lmed & Linear & $-0,58+48,45$ Lmed & 58 & 0,3370 & 1581,87 \\
\hline Eq4 & CLmax & Linear & $0,96+0,52$ CLmax & 58 & 0,8531 & 350,58 \\
\hline Eq5 & CLmed & Linear & $1,30+0,92$ CLmed & 58 & 0,9565 & 103,83 \\
\hline Eq6 & $\mathrm{C}$ & Exponencial & $9,98 \mathrm{e}^{0,03 \mathrm{C}}$ & 58 & 0,9470 & 1269,6 \\
\hline Eq7 & Lmax & Exponencial & $6,60 \mathrm{e}^{0,05 \operatorname{Lmax}}$ & 58 & 0,9218 & 1873,6 \\
\hline Eq8 & Lmed & Exponencial & $6,58 \mathrm{e}^{0,06 \mathrm{Lmed}}$ & 58 & 0,9376 & 1495,3 \\
\hline Eq9 & CLmax & Exponencial & $8,86 \mathrm{e}^{0,04 \mathrm{CLmax}}$ & 58 & 0,9796 & 489,2 \\
\hline Eq10 & CLmed & Exponencial & $10,30 \mathrm{e}^{0,03 \text { CLmed }}$ & 58 & 0,9787 & 510,5 \\
\hline
\end{tabular}

*Variáveis dimensionais lineares: comprimento $(\mathrm{C})$ e largura (L). 
TABELA 2: Coeficiente de correlação das equações que estabelecem as relações entre a área foliar real (AFR) em $\mathrm{cm}^{2}$ e as áreas estimadas do capim-xaraés.

\begin{tabular}{|c|c|c|c|c|c|c|c|c|c|c|c|}
\hline & AFR & Eq1 & $\mathbf{E q 2}$ & Eq3 & $\mathrm{Eq4}$ & Eq5 & Eq6 & Eq7 & Eq8 & Eq9 & Eq10 \\
\hline \multirow[t]{2}{*}{ AFR } & 1,000 & 0,948 & 0,812 & 0,884 & 0,982 & 0,998 & 0,952 & 0,812 & 0,884 & 0,967 & 0,991 \\
\hline & & $<0,05$ & $<0,05$ & $<0,05$ & $<0,05$ & $<0,05$ & $<0,05$ & $<0,05$ & $<0,05$ & $<0,05$ & $<0,0$ \\
\hline \multirow[t]{2}{*}{ Eq1 } & & 1,000 & 0,656 & 0,712 & 0,945 & 0,948 & 0,998 & 0,656 & 0,712 & 0,915 & 0,931 \\
\hline & & & $<0,05$ & $<0,05$ & $<0,05$ & $<0,05$ & $<0,05$ & $<0,05$ & $<0,05$ & $<0,05$ & $<0,05$ \\
\hline \multirow[t]{2}{*}{ Eq2 } & & & 1,000 & 0,877 & 0,857 & 0,803 & 0,658 & 1,000 & $\mathbf{0 , 8 7 7}$ & 0,842 & 0,795 \\
\hline & & & & $<0,05$ & $<0,05$ & $<0,05$ & $<0,05$ & $<0,05$ & $<0,05$ & $<0,05$ & $<0,05$ \\
\hline \multirow[t]{2}{*}{ Eq3 } & & & & 1,000 & 0,843 & $\mathbf{0 , 8 8 8}$ & 0,713 & $\mathbf{0 , 8 7 7}$ & 1,000 & 0,821 & 0,876 \\
\hline & & & & & $<0,05$ & $<0,05$ & $<0,05$ & $<0,05$ & $<0,05$ & $<0,05$ & $<0,05$ \\
\hline \multirow[t]{2}{*}{$\mathrm{Eq} 4$} & & & & & 1,000 & 0,979 & 0,949 & $\mathbf{0 , 8 5 7}$ & 0,843 & 0,987 & 0,972 \\
\hline & & & & & & $<0,05$ & $<0,05$ & $<0,05$ & $<0,05$ & $<0,05$ & $<0,05$ \\
\hline \multirow[t]{2}{*}{ Eq5 } & & & & & & 1,000 & 0,952 & 0,803 & $\mathbf{0 , 8 8 8}$ & 0,963 & 0,994 \\
\hline & & & & & & & $<0,05$ & $<0,05$ & $<0,05$ & $<0,05$ & $<0,05$ \\
\hline \multirow[t]{2}{*}{ Eq6 } & & & & & & & 1,000 & 0,658 & 0,712 & 0,927 & 0,942 \\
\hline & & & & & & & & $<0,05$ & $<0,05$ & $<0,05$ & $<0,05$ \\
\hline \multirow[t]{2}{*}{ Eq7 } & & & & & & & & 1,000 & $\mathbf{0 , 8 7 7}$ & 0,842 & 0,795 \\
\hline & & & & & & & & & $<0,05$ & $<0,05$ & $<0,05$ \\
\hline \multirow[t]{2}{*}{ Eq8 } & & & & & & & & & 1,000 & $\mathbf{0 , 8 2 1}$ & 0,876 \\
\hline & & & & & & & & & & $<0,05$ & $<0,05$ \\
\hline \multirow[t]{2}{*}{ Eq9 } & & & & & & & & & & 1,000 & 0,973 \\
\hline & & & & & & & & & & & $<0,05$ \\
\hline Eq10 & & & & & & & & & & & 1,000 \\
\hline
\end{tabular}

Valores abaixo de cada coeficiente referem-se ao nível de significância $\mathrm{P}>1 \frac{1}{2} \mathrm{t}^{1} / 2$.

Para o capim-massai, as equações que melhor estimaram a AF por terem apresentado os melhores valores de $r^{2}$ e dos coeficientes de correlação foi a equação linear Eq5 (1,30+0,92 CLmed) e as exponenciais Eq9 $\left(8,86 \mathrm{e}^{0,04 C \operatorname{lmax}}\right)$ e Eq10 $\left(10,30 \mathrm{e}^{0,03 C L \operatorname{lm} d}\right)$ representadas na Figura 2.
Ao considerarmos a soma de quadrados do resíduo estas três equações também apresentaram os menores valores, sendo respectivamente iguais a 103,83 , 489,2 e 510,5. De forma análoga, Bianco et al. (2001) concluiram que o produto comprimento $\mathrm{x}$ largura, de folhas de Panicum maximum gerou a melhor equação na estimativa da área foliar real desta espécie. 
TABELA 3: Coeficiente de correlação das equações (Eq) que estabelecem as relações entre a área foliar real (AFR) em $\mathrm{cm}^{2}$ e as áreas estimadas do capim-massai.

\begin{tabular}{|c|c|c|c|c|c|c|c|c|c|c|c|}
\hline & AFR & Eq1 & Eq2 & Eq3 & Eq4 & Eq5 & Eq6 & Eq7 & Eq8 & Eq9 & Eq10 \\
\hline \multirow[t]{2}{*}{ AFR } & 1,000 & 0,785 & 0,468 & $\mathbf{0 , 5 8 9}$ & 0,924 & 0,978 & 0,728 & 0,469 & $\mathbf{0 , 5 9 0}$ & 0,912 & 0,965 \\
\hline & & $<0,05$ & $<0,05$ & $<0,05$ & $<0,05$ & $<0,05$ & $<0,05$ & $<0,05$ & $<0,05$ & $<0,05$ & $<0,05$ \\
\hline \multirow[t]{2}{*}{ Eq1 } & & 1,000 & 0,033 & 0,034 & 0,855 & 0,795 & 0,948 & 0,033 & 0,035 & 0,781 & 0,748 \\
\hline & & & $>0,05$ & $>0,05$ & $<0,05$ & $<0,05$ & $<0,05$ & $>0,05$ & $>0,05$ & $<0,05$ & $<0,05$ \\
\hline \multirow[t]{2}{*}{ Eq2 } & & & 1,000 & 0,662 & 0,529 & 0,423 & 0,035 & 1,000 & 0,662 & 0,543 & 0,444 \\
\hline & & & & $<0,05$ & $<0,05$ & $<0,05$ & $>0,05$ & $<0,05$ & $<0,05$ & $<0,05$ & $<0,05$ \\
\hline \multirow[t]{2}{*}{ Eq3 } & & & & 1,000 & 0,392 & 0,619 & 0,038 & 0,662 & 1,000 & 0,454 & 0,645 \\
\hline & & & & & $<0,05$ & $<0,05$ & $>0,05$ & $<0,05$ & $<0,05$ & $<0,05$ & $<0,05$ \\
\hline \multirow[t]{2}{*}{ Eq4 } & & & & & 1,000 & 0,909 & 0,802 & 0,529 & 0,393 & 0,958 & $\mathbf{0 , 8 8 4}$ \\
\hline & & & & & & $<0,05$ & $<0,05$ & $<0,05$ & $<0,05$ & $<0,05$ & $<0,05$ \\
\hline \multirow[t]{2}{*}{ Eq5 } & & & & & & 1,000 & 0,752 & 0,424 & 0,619 & 0,902 & 0,989 \\
\hline & & & & & & & $<0,05$ & $<0,05$ & $<0,05$ & $<0,05$ & $<0,05$ \\
\hline \multirow[t]{2}{*}{ Eq6 } & & & & & & & 1,000 & 0,035 & 0,039 & 0,792 & 0,732 \\
\hline & & & & & & & & $>0,05$ & $>0,05$ & $<0,05$ & $<0,05$ \\
\hline \multirow[t]{2}{*}{ Eq7 } & & & & & & & & 1,000 & 0,662 & 0,543 & 0,444 \\
\hline & & & & & & & & & $<0,05$ & $<0,05$ & $<0,05$ \\
\hline \multirow[t]{2}{*}{ Eq8 } & & & & & & & & & 1,000 & 0,455 & 0,646 \\
\hline & & & & & & & & & & $<0,05$ & $<0,05$ \\
\hline \multirow[t]{2}{*}{ Eq9 } & & & & & & & & & & 1,000 & 0,915 \\
\hline & & & & & & & & & & & $<0,05$ \\
\hline Eq10 & & & & & & & & & & & 1,000 \\
\hline
\end{tabular}

Valores abaixo de cada coeficiente referem-se ao nível de significância $\mathrm{P}>1 \frac{1}{2} \mathrm{t}^{1} \frac{2}{2}$.

FIGURA 1: Relação entre a área foliar real (AFR) e a área resultante do comprimento x largura média das folhas em capim-xaraés.

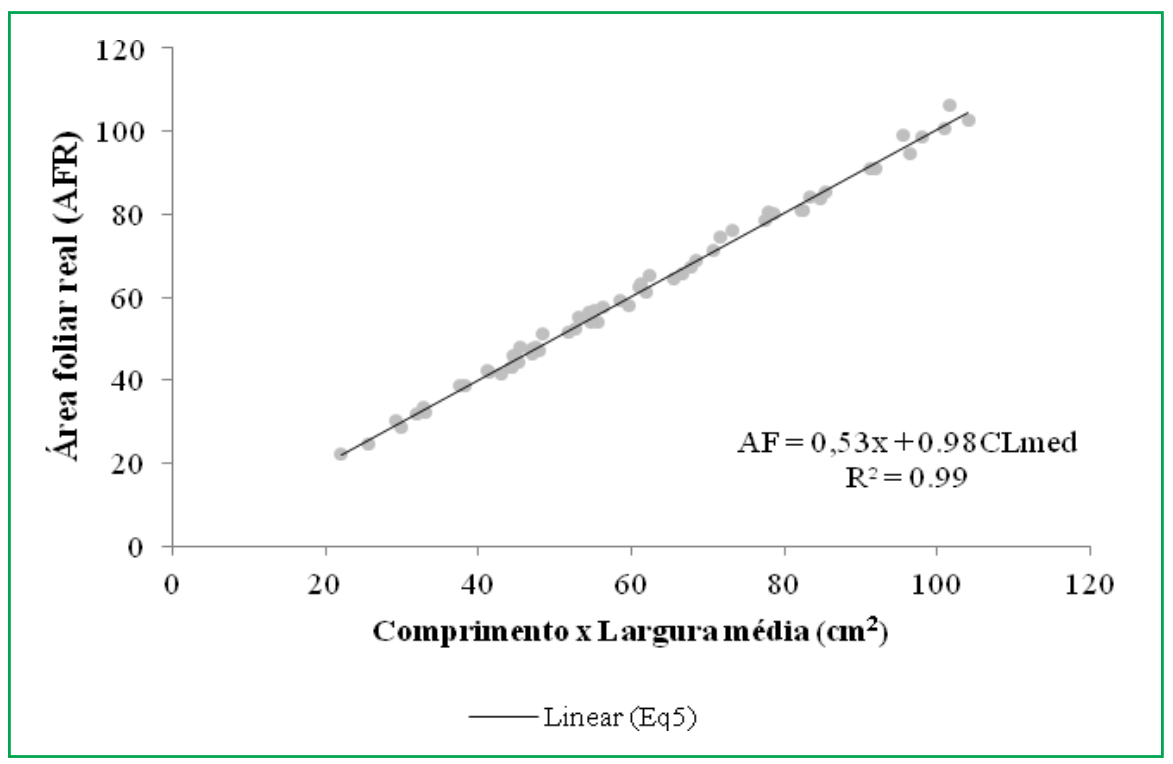


FIGURA 2: Relação entre a área foliar real (AFR) e a área resultante do comprimento x largura das folhas em capim-massai.

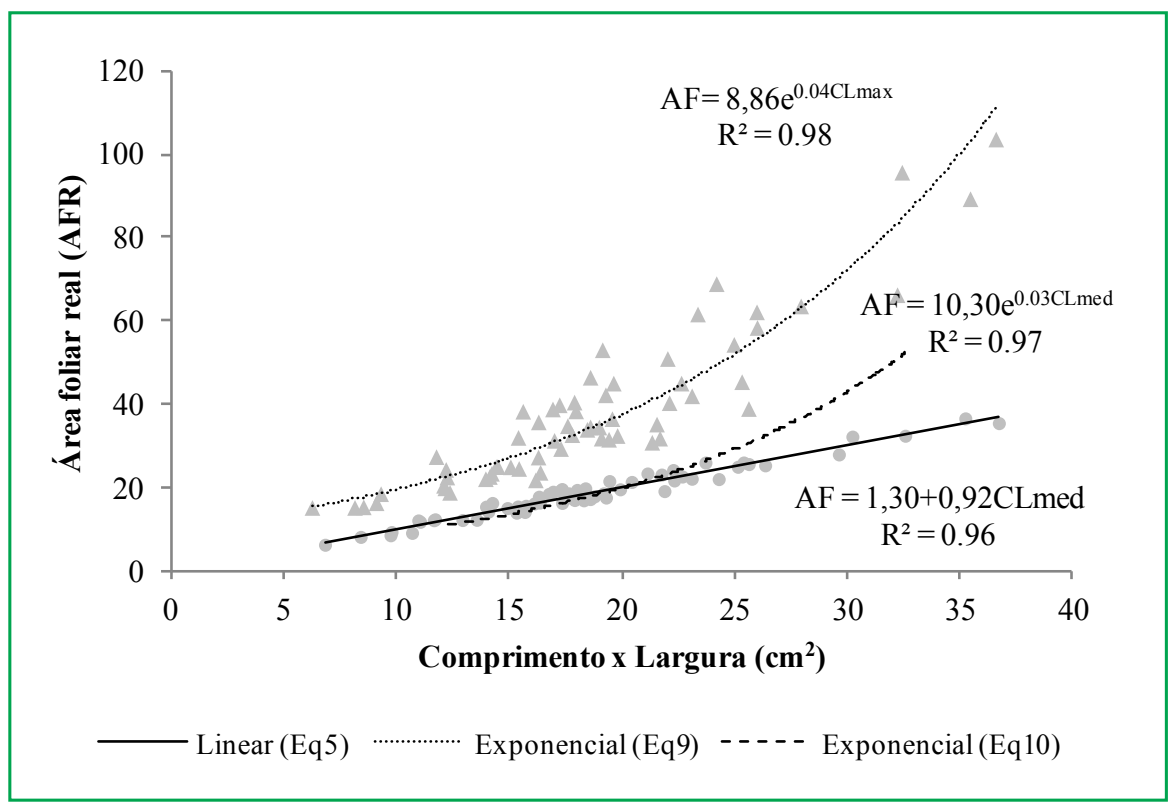

Ao analisar a praticidade de qualquer método de pesquisa, este deve ser selecionado por um pesquisador, sempre considerando a disponibilidade de recursos materiais, humanos e financeiros, além de tempo para realizar as medidas e/ou coletas com a precisão desejada. No caso específico do uso de medidas lineares do limbo foliar para estimativas de área foliar de espécies forrageiras, embora a utilização de ambas as medidas, comprimento e largura do limbo da folha, seja significativamente mais precisa que o uso de apenas uma dimensão, tal procedimento requer duas vezes o número de medidas a serem tomadas (SILVA et al., 2002). Assim, se houver necessidade de realizar as medições mais rapidamente com redução de $50 \%$ no número de medições, e com boa precisão, tanto para o capim-xaraés quanto para o capim-massai, a dimensão comprimento $(\mathrm{C})$ pode ser uma alternativa viável para ambas as espécies, representadas para os dois capins, pelas equações Eq6, 26,44e $\mathrm{e}^{0,01 \mathrm{C}}\left(\mathrm{r}^{2}=0,9830\right.$ e $\mathrm{R}=0,952$ ) e $9,98 \mathrm{e}^{0,03 \mathrm{C}}\left(\mathrm{r}^{2}=0,9470\right.$ e $\left.\mathrm{R}=0,728\right)$ respectivamente.

Ao se comparar o método de medidas lineares com o uso de régua graduada ao método do Li-Cor ${ }^{\mathbb{R}}$, pode-se afirmar que as medidas de área de folhas de gramíneas, geradas por ambos os métodos são muito próximas. $\mathrm{O}$ grande diferencial entre os dois métodos, porém, está no custo que os envolve.
A partir dos resultados, foi possível concluir que as equações lineares e exponenciais estudadas podem ser utilizadas para estimar a área foliar real do capim-xaraés, porém, ter-se-á maior precisão utilizando-se a equação linear 0,53+0,98 CLmed. As equações que estimam de maneira mais confiável a área foliar individual do capimmassai é a linear 1,30+0,92 CLmed e as exponenciais $8,86 \mathrm{e}^{0,04 C l m a x}$ e $10,30 \mathrm{e}^{0,03 C l m e d}$. Além disso, as estimativas de área foliar de capim-xaraés e capim-massai por meio de medidas simples de comprimento e largura de folhas são eficientes e precisas, com baixo custo para as realizações.

\section{Referências}

ADAMI, M.; HASTENREITER, F. A.; FLUMIGNAN, D. L.; FARIA, R. T. Estimativa de área de folíolos de soja usando imagens digitais e dimensões foliares. Bragantia, Campinas, v. 67, p. $1053-$ 1058, 2008.

BIANCO, S.; BRENDOLAN, R. A.; ALVES, P. L. da C. A.; PITELLI, R. A. Estimativa da área foliar de Brachiaria decumbens Stapf. e Brachiaria brizantha (Hochst.) Stapf. Planta Daninha, Viçosa, v. 18, p. 79-83, 2000.

BIANCO, S.; CARVALHO, L. B.; BIANCO, M. S. Estimativa da área foliar de sida cordifolia e sida rhombifolia usando dimensões lineares do limbo foliar. Planta Daninha, Viçosa, v. 26, p. 807-813, 2008.

BIANCO, S.; PITELLI, R.; PERECIN, D. Estimativa da área foliar de Panicum maximum usando dimensões lineares do limbo foliar. Planta Daninha, Viçosa, v. 19, p. 217-221, 2001. 
CARVALHO, L. B.; BIANCO, S.; GALATI, V. C.; PANOSO, A. R. Determination of Merremia cissoides leaf área using the linear measures of the leaflets. Acta Scientiarum Agronomy, Maringá, v. 33, p. 473-476, 2011.

EMBRAPA. Capim-massai (Panicum maximum cv. Massai): alternativa para diversificação de pastagens. Campo Grande: Embrapa Gado de Corte, 2011. 5 p. (Comunicado Técnico, 69)

EUCLIDES, V. P. B.; MACEDO, M. C. M.; VALLE, C. B.; DIFANTE, G, S.; BARBOSA, R. A.; CACERE, E, R. Valor nutritivo da forragem e produção animal em pastagens de Brachiaria brizantha. Pesquisa Agropecuária Brasileira, Brasília, v. 44, p. 98-106, 2009.

GALZERANO, L.; MALHEIROS, E. B; MORGADO, E. da S.; SILVA, W. L.; COSTA, J. P. R.; CAPUTTI, G. de P.; RUGGIERI, A. C. Medidas lineares na estimativa da área foliar do capimaruana. Nucleus Animalium, Ituverava, v. 4, p. 78-82, 2012.
MALDANER, I. C.; HELDWEIN, A, B.; LOOSE, L. H.; LUCAS, D. D. P.; GUSE, F. I.; BORTOLUZZI, M. P. Modelos de determinação não-destrutiva da área foliar em girassol. Ciência Rural, Santa Maria, v. 39, p. 1356-1361, 2009.

SAS. Statistical Analysis Systems user's guide: Stat - Version 9. Cary: SAS, 2002. 1464 p.

SILVA, L. C.; SANTOS, J. W.; VIEIRA, D. J.; BELTRÃO, N. E. M.; ALVES, I.; JERÔNIMO, J. F. Um método simples para se estimar área foliar de plantas de gergelim (Sesamum indicum 1.). Revista brasileira oleaginosas e fibrosas, Campina Grande, n. 6, p. 491-496, 2002.

VALLE, C. B.; EUCLIDES, V. P. B.; PEREIRA, J. M.; VALERIO, J. R.; PAGLIARINI, M. S.; MACEDO, M. C. M.; LEITE, G. G.; LOURENÇO, A. J.; FERNANDES, C. D.; FILHO, M. B. D.; LEMPP, B.; POTT, A.; SOUZA, M. A. O capim-xaraés (Brachiaria brizantha cv. Xaraés) na diversificação de pastagens de braquiária. Campo Grande: Embrapa Gado de Corte, 2004. 36 p. (Embrapa Gado de Corte. Documentos, 149) 